\title{
PLAGAS Y ENFERMEDADES EN PLANTACIONES DE TECA (Tectona grandis L.F) EN LA ZONA DE BALZAR, PROVINCIA DEL GUAYAS
}

\author{
Tonny Flores Velasteguí ${ }^{1},{ }^{\wedge}$ Rommel Crespo Gutiérrez ${ }^{2}$ y Fernando Cabezas Guerrero ${ }^{2}$ \\ ${ }^{1}$ Departamento de protección forestal. TROPIBOSQUES S.A. $1.5 \mathrm{Km}$ vía Balzar - El Empalme. \\ Hacienda "La Igea". Balzar - Guayas - Ecuador \\ ${ }^{2}$ Unidad de Investigación Cientifica y Tecnológica, Universidad Técnica Estatal de Quevedo, km 7 vía \\ Quevedo - El Empalme, C. P. 73. Mocache,Los Ríos, Ecuador. ${ }^{\circ}$ rscgtexcmex@hotmail.com \\ RESUMEN
}

Ce diagnosticó las plagas y enfermedades en planta$\checkmark$ ciones de Tectona grandis L.F (Teca) en la zona de Balzar, provincia del Guayas. Los objetivos fueron recolectar e identificar los insectos plagas y los agentes causales de enfermedades que se encuentran presentes en las plantaciones de Teca, determinar el nivel de incidencia y severidad de los problemas fitosanitarios. Se realizaron muestreos al azar mediante recorridos en transeptos y se establecieron parcelas de $500 \mathrm{~m}^{2}$ distribuidas según las superficies de las haciendas. Los insectos se recolectaron en época seca y lluviosa durante seis meses. Las plagas y enfermedades de mayor incidencia y severidad fueron: Hyadaphis erysimi y Hortensia similis (Homóptera); y Colletotrichum sp., Olivea tectonae y Ceratocystis sp. en época lluviosa. Atta sp. (Hymenóptera), Hemileuca maia y Scolytus sp. (Coleóptera); y Olivea tectonae en época seca.

Palabras claves: Plagas, enfermedades, Tectona grandis L.F, insectos.

\section{INTRODUCCIÓN}

$\mathrm{L}^{\mathrm{a}}$ Teca es un árbol caducifolio de tamaño grande, natural del sudeste de Asia, en donde alcanza $45 \mathrm{~m}$ de altura y desarrolla un tronco con contrafuertes al llegar a la madurez. La Teca, fuente de una de las maderas tropicales más valiosas y mejor conocidas, ha sido plantada extensamente para la producción de madera para la construcción naviera, muebles y carpintería en general (Weaver, 2000). Se estima que existen 12,000 has de Teca en el Ecuador, las cuales se encuentran distribuidas en el Litoral ecuatoriano (OIMT, 2004).

La mayoría de los patógenos de la Teca han sido identificados en la India y el Lejano Oriente, con sólo unos cuantos registrados en plantaciones en África, América y en áreas lejos de su región nativa. A pesar de esto, existe muy poca información disponible acerca de sus consecuencias económicas (Gibson, 1975 citado por Weaver, 2000).

Los árboles, como cualquier otra planta, son susceptibles de ataque de organismos fitófagos que pueden llegar a comprometer seriamente su sobrevivencia, visto desde un punto de vista netamente económico pueden causar un detrimento importante en la productividad y valor de los productos que se espera obtener de

Recibido: Enero, 2009. Aceptado: Diciembre, 2009.

Publicado como ARTÍCULO en Ciencia y Tecnología 3(1): 15-22. 2010
Tt was diagnosed the pest and diseases in Tectona grandis L.F (Teak) plantations in the area of Balzar, Guayas province. The objectives were to collect and to identify the insects plagues and the causal agents of illnesses that are present in the Teak plantations, to determine the level of incidence and severity of the fitosanitary problems. They were carried out samplings at random by means of journeys in transepts and parcels of $500 \mathrm{~m}^{2}$ distributed according to the surfaces of the country properties settled down. The insects were collected in dry and rainy season during six months. The pest and diseases of more incidence and severity were: Hyadaphis erysimi and Hortensia similis (Homoptera); and Colletotrichum sp., Olivea tectonae and Ceratocystis sp. in rainy season. Atta sp. (Hymenoptera), Hemileuca maia Drury and Scolytus sp. (Coleoptera); and Olivea tectonae in dry season.

Key words: Pest, diseases, Tectona grandis L.F, insects.

la especie. Los árboles pueden sufrir daños estando en ambientes naturales, en plantaciones puras o en sistemas agrosilvopastoriles y agroforestales. Por su extensión y distribución las plantaciones forestales puras son un medio muy propicio para el desarrollo epidémico de problemas fitosanitarios, máxime si durante su establecimiento y desarrollo no se toman las medidas preventivas apropiadas. De acuerdo con su origen geográfico, los organismos causantes de problemas fitosanitarios pueden ser nativos o introducidos; los que a su vez pueden ser específicos ó tener un rango amplio o reducido de hospedantes. Más aún, algunos están en la capacidad de colonizar y adaptarse a nuevos hospedantes cuando la oferta alimenticia es favorable (Pinzón, 2007).

Los problemas fitosanitarios representan una amenaza para los productos forestales, por lo que el desarrollo de conocimientos en este campo y la difusión de los mismos, es fundamental dentro de la silvicultura de plantaciones, y específicamente para la Teca (Arguedas, 2003).

Se considera a un insecto plaga forestal, cuando como consecuencia de sus daños, se producen pérdidas que afectan los valores ecológicos, económicos y sociales que se relacionan con los árboles forestales y de sombra (Coulson y Witter, 1990 citados por Pinzón, 1997). 
Se reconoce como una enfermedad a todo cambio o alteración morfo-fisiológica, con suficiente duración e intensidad para causar perjuicio o cesación de la actividad vital. En una aceptación más amplia, se puede considerar como un disturbio en la estructura y funciones normales de la planta, afectando al desarrollo y calidad (Ramírez, 1997).

Los objetivos de este estudio fueron recolectar insectos y tejidos vegetales enfermos para identificar las plagas y los agentes causales de enfermedades, para poder determinar el nivel e incidencia de los problemas fitosanitarios.

\section{Materiales y Métodos}

$\mathrm{L}$ a investigación se realizó en las haciendas: "Las Piedras" de 250 ha, "Alianza" de 88 ha, "Cerro de Hojas" de 350 ha, "Hacha" con 800 ha, pertenecientes a Tropibosques S.A, localizadas en el cantón Balzar, se encuentran a $40 \mathrm{msnm}$, presenta una precipitación media anual de $1,222 \mathrm{~mm}$, una temperatura promedio anual de $24.4^{\circ} \mathrm{C}$, una humedad relativa de $72.9 \%$, una heliofanía media anual de 881 horas luz y un tipo de suelo arcilloso-arenoso con un $\mathrm{pH}$ 5.9-7.1. Las plantaciones de Teca fueron de 1 a 5 años de edad sembradas a un espaciamiento de $3 \times 3 \mathrm{~m}$.

Se realizó un muestreo al azar en 11.35 has ( $1 \%$ del total de has plantadas) y se establecieron 200 parcelas de $500 \mathrm{~m}^{2}$. Todos los árboles de las parcelas fueron evaluados siendo en total 10,600 árboles los evaluados (53 por parcela). Se registraron los datos de plagas y enfermedades tanto en época seca como en época lluviosa por seis meses.

Para registrar los datos de los insectos plagas mediante la observación directa en el campo se utilizó un formulario previamente elaborado. La identificación de los insectos la realizaron especialistas en entomolo- gía directamente en el campo, los especimenes de los insectos de menor tamaño se los recolectó en frascos entomológicos con alcohol al $70 \%$ para su posterior identificación.

Para identificar las enfermedades, se recolectaron muestras de hojas, raíces, pedazos de madera y partes apicales que presentaron síntomas de enfermedades, las mismas que se las colocó en fundas plásticas y de papel debidamente etiquetadas con información básica como nombre del colector, parte del árbol donde se efectuó la toma de muestra, ubicación geográfica del árbol, edad de la plantación, fecha y hora. Las muestras de hojas se envolvieron en papel periódico humedecido para que mantengan la humedad y luego se la refrigeró por un lapso de 48 horas. La identificación de los agentes causales de las enfermedades en las muestras se lo realizó en los laboratorios de fitopatología de las estaciones Pichilingue y Boliche, pertenecientes al Instituto Nacional de Investigaciones Agropecuarias (INIAP).

Para establecer la severidad de los insectos plagas se utilizó la escala modificada propuesta por el Ministerio de Agricultura y Ganadería (MAG, 1986):

$0=$ Ausencia

$1=$ Leve $o$ esporádico

$2=$ Moderado

$3=$ Fuerte

Para categorizar los problemas fitopatológicos se empleó la escala usada por Morales y Niessen (1988), citados por Gómez (1999):

$0=$ Ausencia

$1=$ Síntomas ligeros

$2=$ Síntomas moderados

3 = Síntomas severos

Empleando las escalas propuestas se procedió a caracterizar la incidencia y severidad de los insectos plagas y enfermedades en plantaciones de Teca, empleándose índices arbitrarios de acuerdo al daño causado en el árbol (Cuadros 1 y 2).

Cuadro 1. Índices arbitrarios utilizados para determinar la incidencia de los insectos plaga

\begin{tabular}{ccccc}
\hline \multirow{2}{*}{ Insectos Plagas } & \multirow{2}{*}{ Indicador } & \multicolumn{3}{c}{ Incidencia } \\
\cline { 3 - 4 } & & Leve & Moderado & Fuerte \\
\hline Aphydidae & Individuos & $1-$ Oct & $20-$ Nov & $>20$ \\
Coreidae & Individuos & 1 -May & $6-$ Oct & $>10$ \\
Cicadellidae & Individuos & $1-$ May & $6-$ Oct & $>10$ \\
Scolytidae & Individuos & $1-$ May & $6-$ Oct & $>10$ \\
Scarabaeidae & Individuos & $1-$ May & $6-$ Oct & $>10$ \\
Formicidae & Árbol defoliado & $0-25 \%$ & $26-50 \%$ & $>50 \%$ \\
Saturniidae & Árbol defoliado & $0-25 \%$ & $26-50 \%$ & $>50 \%$ \\
Aleyroidae & Área foliar afectada & $0-25 \%$ & $26-50 \%$ & $>50 \%$ \\
Termitidae & Daño en corteza & $0-25 \%$ & $26-50 \%$ & $>50 \%$ \\
\hline
\end{tabular}


Cuadro 2. Índices arbitrarios utilizados para determinar las enfermedades en árboles de Teca

\begin{tabular}{ccccc}
\hline \multirow{2}{*}{ Enfermedad } & \multirow{2}{*}{ Indicador } & \multicolumn{3}{c}{ Síntomas } \\
\cline { 3 - 5 } & & Leve & Moderado & Severo \\
\hline Ceratocystis sp. & Muerte descendente & $0-25 \%$ & $26-50 \%$ & $100 \%$ \\
Phythopthora spp. & Muerte ascendente & $0-25 \%$ & $26-50 \%$ & $100 \%$ \\
Colletotrichum sp. & Área foliar afectada & $0-25 \%$ & $26-50 \%$ & $>50 \%$ \\
Olivea tectonae (Roya) & Área foliar afectada & $0-25 \%$ & $26-50 \%$ & $>50 \%$ \\
\hline
\end{tabular}

Para transformar los datos obtenidos de severidad de insectos plagas y enfermedades a porcentajes se utilizó la ecuación propuesta por Towsend y Heuberger (1943) citados por Rodríguez et al. (2006) que se describe a continuación:

$$
\% S=a \cdot b / N K * 100
$$

Donde: $\% S=$ Porcentaje de severidad; $a=$ Plantas; $b=$ Categoría de daño de planta; $N=$ Total de plantas y $K=$ Categoría mayor

\section{Resultados}

Insectos plagas en época lluviosa

Se identificaron insectos de los ordenes Coleóptera, Hemíptera, Hymenóptera, Homóptera e Isóptera, los mismos que causaron daño en la raíz, corteza, xilema y en el follaje de árboles de Teca (Cuadro 3).

\section{Incidencia de insectos plagas en época lluviosa}

Los insectos con mayor incidencia en las cuatro haciendas fueron los áfidos o pulgones Hyadaphis erysimi (Homóptera, Aphydidae) que no causaron mayores daños en el follaje, Hortensia similis (Homóptera, Cicadellidae) la cual no evidenció daños de importancia en el follaje, Phyllophaga spp. (Coleóptera, Scarabaeidae) que causo daños de moderada consideración (Figura 1).

Cuadro 3. Insectos plaga registrados en plantaciones de Teca de la empresa Tropibosques S.A, durante la época lluviosa del año 2004

\begin{tabular}{ccccc}
\hline Orden & Familia* & Nombre científico & Nombre común & Tipo de daño \\
\hline Coleóptera & Buprestidae & Chrysobothis femorata Oliv. & & Barrena el xilema \\
Coleóptera & Scarabaeidae & Phyllophaga spp. & Orozco & Comen la raíz \\
Coleóptera & Scolytidae & Scolytus sp. & Scolitidos o polillas & Perforan la corteza \\
Hemíptera & Coreidae & Anasa sp. & Chinches & Picador - chupador \\
Hemíptera & Pentatomidae & Nesara sp. & Chinches & Picador - chupador \\
Hymenóptera & Formicidae & Atta sp. & Hormiga arriera & Defoliador \\
Homóptera & Aphydidae & Hyadaphis erysimi & Pulgones o áfidos & Picador - chupador \\
Homóptera & Cicadellidae & Oncomotepia sp. & Camina de lado & Picador - chupador \\
Homóptera & Cercopidae & Aeneolamia postica & Salivazo & Chupador de savia \\
Homóptera & Cicadellidae & Hortensia similis & Cigarrita & Chupador de savia \\
Isóptera & Termitidae & & Termitas & Barrena la corteza \\
\hline
\end{tabular}

* Identificación a nivel de familia por el Dr. R. Wills Flowers, Professor Center for Biological Control Florida A M University 


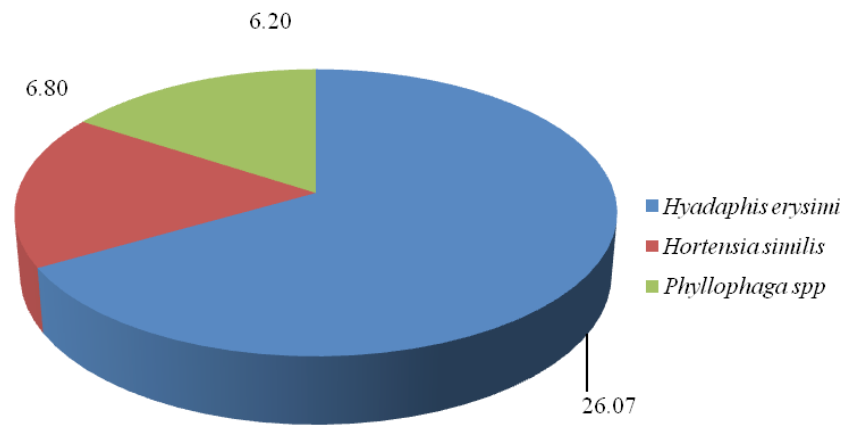

Figura 1. Porcentaje de incidencia de insectos plagas durante la época lluviosa del año 2004 en cuatro haciendas de la empresa Tropibosques S.A.

Insectos plagas en época seca

Se registraron insectos de los órdenes Homóp- tera, Hymenóptera, Coleóptera, Isóptera, Lepidóptera y Orthóptera, causando daños en el follaje, corteza, duramen, brotes, yemas de árboles de Teca (Cuadro 4).

Cuadro 4. Insectos plagas registrados en plantaciones de teca de la empresa Tropibosques S.A durante la época seca del año 2004

\begin{tabular}{ccccc}
\hline Orden & Familia* & Nombre científico & Nombre común & Tipo de daño \\
\hline Homóptera & Aphydidae & Hyadaphis erysimi & Pulgones o áfidos & Picador - chupador \\
Homóptera & Aleyrodidae & Bemisia tabaci & Mosca blanca & Chupador de savia \\
Hymenóptera & Formicidae & Atta sp. & Hormiga arriera & Defoliador \\
Coleóptera & Scolytidae & Scolytus sp. & Scolitidos o polillas & Perforan la corteza \\
Coleóptera & Cerambicidae & Oberea tripunctata & Barrenador de la teca & Barrena el duramen \\
Isóptera & Termitidae & & Termitas & Barrena la corteza \\
Lepidóptera & Saturniidae & Hemileuca maia Drury & Defoliador de la teca & Defoliador \\
Orthóptera & Acrididae & Schistocercu spp. & Grillos & Trozan brotes y yemas \\
\hline
\end{tabular}

* Identificación a nivel de familia por el Dr. R. Wills Flowers, Professor Center for Biological Control Florida A M University

Incidencia de insectos plagas en época seca

Las plagas de mayor incidencia en las cuatro haciendas evaluadas en esta época fueron orugas defoliadoras Hemileuca maia Drury (Lepidóptera, Saturniidae), hormiga arriera Atta sp. (Hymenoptera, Formici- dae), y escolitidos Scolytus sp. (Coleóptera, Scolytidae) perforadores de corteza (Figura 2). Los daños por los defoliadores Atta sp. y Hemileuca maia fueron leves, a excepción de algunas plantas que mostraron daños severos.

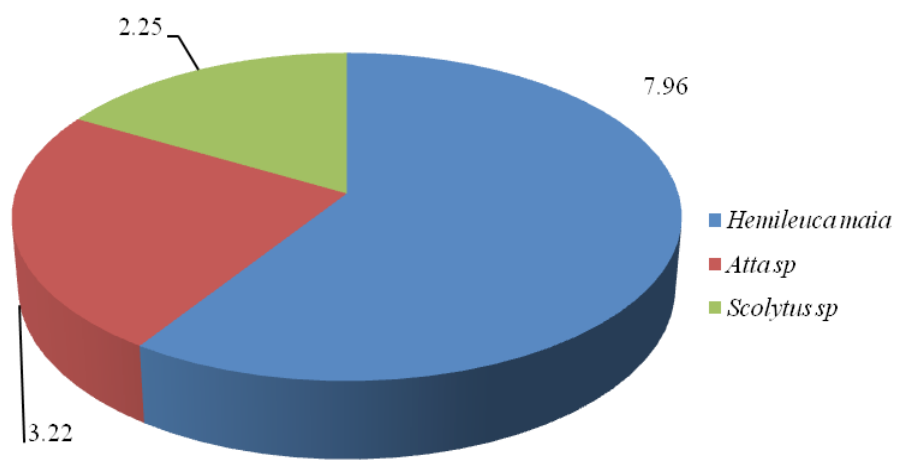

Figura 2. Porcentaje de incidencia de insectos plagas durante la época seca del año 2004 en cuatro haciendas de la empresa Tropibosques S.A. 


\section{Enfermedades época lluviosa}

Se detectaron enfermedades causadas por hongos que afectan al follaje y al sistema vascular y radicular (Cuadro 5). Las enfermedades con mayor relevancia fueron las detectadas en el follaje de plantaciones y vivero, especialmente la causada por Colletotrichum sp. En árboles que se encontraban en suelos anegados se detectó el patógeno Phythopthora spp. que causa la pudrición de la raíz principalmente de las secundarias, causando la muerte descendente del árbol. En árboles de diferentes edades se observó un marchitamiento desde el brote terminal avanzando hacia abajo del tallo causado por Ceratocystis sp.

Cuadro 5. Hongos patógenos registrados en plantaciones de Teca de la empresa Tropibosques S. A. durante la época lluviosa del año 2004

\begin{tabular}{cccc}
\hline Clase & Orden & Nombre científico* & Tipo de daño \\
\hline Deuteromycetes & Melanconiales & Colletotrichum sp. & Marchitamiento del follaje \\
Basidiomycetes & Uredinales & Olivea tectonae & Marchitamiento del follaje \\
Ascomycetes & & Ceratocystis sp. & Daño al sistema vascular \\
Oomycetes & Peronosporales & Phytophthora spp. & Daño al sistema radicular \\
\hline
\end{tabular}

* Enfermedades identificadas por el Departamento de Protección Vegetal de las estaciones experimentales Pichilingue y Boliche del INIAP

\section{Incidencia de enfermedades en época lluviosa}

La enfermedad con mayor incidencia en las cuatro haciendas durante la época lluviosa fue Colletotrichum sp. que causó daños leves y moderados a excep- ción de algunos árboles que presentaron daños severos en toda el área foliar. Ceratocystis sp. presentó una menor incidencia pero causo daños moderados y severos en los árboles infectados (Figura 3).

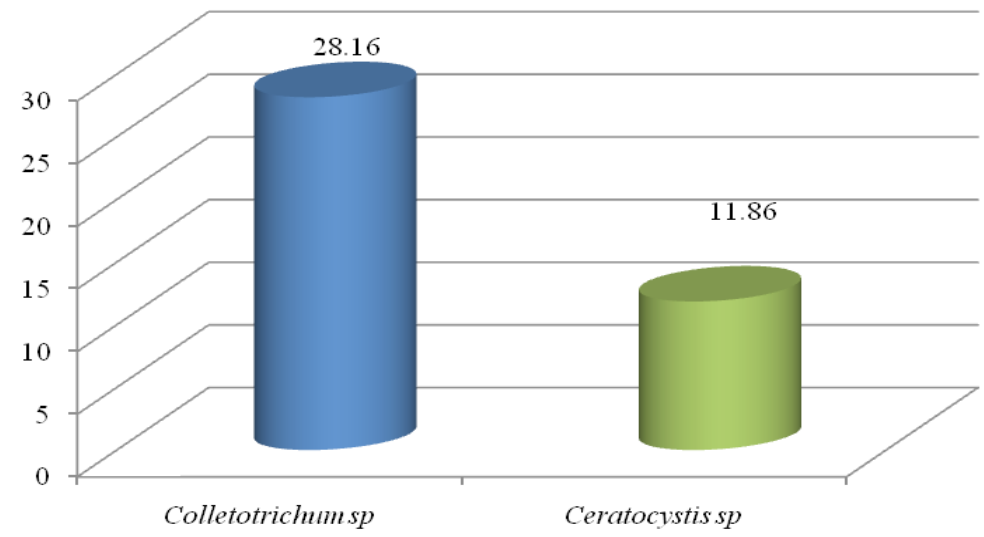

Figura 3. Porcentaje de incidencia de enfermedades causadas por hongos patógenos durante la época lluviosa del año 2004 en cuatro haciendas de la empresa Tropibosques S.A.

\section{Enfermedades en época seca}

Se identificaron enfermedades causadas por hongos que afectaron al follaje, fuste, ápices, ramas, sistema vascular y radicular (Cuadro 6). Se presentaron grandes manchas desde el área apical hasta el área basal del follaje variando de tonalidad de verde claro, amarillo, rojizo a café oscuro causadas por Pastalotia palma- rum. También se detectó otros patógenos de menor incidencia en el follaje como Nigrospora sp., Aspergillus niger y Rizophus estolonifer.

En el fuste, en los puntos de poda, se detectaron exudaciones de color oscuro que presentaban pequeñas aberturas longitudinales, al realizar el corte se observó que el duramen estaba totalmente rodeado de una coloración oscura con bordes café, la misma que 
descendía hasta el cuello del árbol. Esta enfermedad la causa el hongo Botryodiplodia sp., pero no se presentó mortalidad de los árboles infectados.

Se detectó la muerte de ápices y ramas e inclu- so de árboles causadas por Botryodiplodia theobromae, el cual provoca lesiones circulares a ovaladas alargadas de hasta $15 \mathrm{~cm}$ de longitud, de color oscuro que pueden estar cubiertas por grandes masas de esporas.

Cuadro 6. Hongos patógenos registrados en plantaciones de Teca de la empresa Tropibosques S. A. durante la época seca del año 2004

\begin{tabular}{|c|c|c|c|}
\hline Clase & Orden & Nombre científico* & Tipo de daño \\
\hline Deuteromycetes & Melanconiales & Colletotrichum sp. & Marchitamiento del follaje \\
\hline Basidiomycetes & Uredinales & Olivea tectonae & Marchitamiento del follaje \\
\hline \multirow[t]{4}{*}{ Deuteromycetes } & Melanconiales & Pestalotia palmarum & Marchitamiento del follaje \\
\hline & & Nigrospora $\mathrm{sp}$. & Marchitamiento del follaje \\
\hline & & Aspergillus niger & Marchitamiento del follaje \\
\hline & & Rizophus estolonifer & Marchitamiento del follaje \\
\hline Ascomycetes & & Ceratocystis sp. & Daño al sistema vascular \\
\hline Oomycetes & Peronosporales & Phytophthora spp. & Daño al sistema radicular \\
\hline Deuteromycetes & & Botryodiplodia theobromae & Muerte de ápices y ramas \\
\hline Deuteromycetes & & Botryodiplodia sp. & Daño en el fuste \\
\hline
\end{tabular}

* Enfermedades identificadas por el Departamento de Protección Vegetal de las estaciones experimentales Pichilingue y Boliche del INIAP

Incidencia de enfermedades en época seca

Olivea tectonae tuvo una alta incidencia y causo daños severos en el follaje de los árboles infectados, los patógenos con menor incidencia fueron Colle- totrichum sp. que presentó daños leves y Ceratocystis sp. que causo daños severos en los árboles infectados. Phytophthora sp. tuvo una mínima incidencia pero causó daños severos en el sistema radicular (Figura 4).

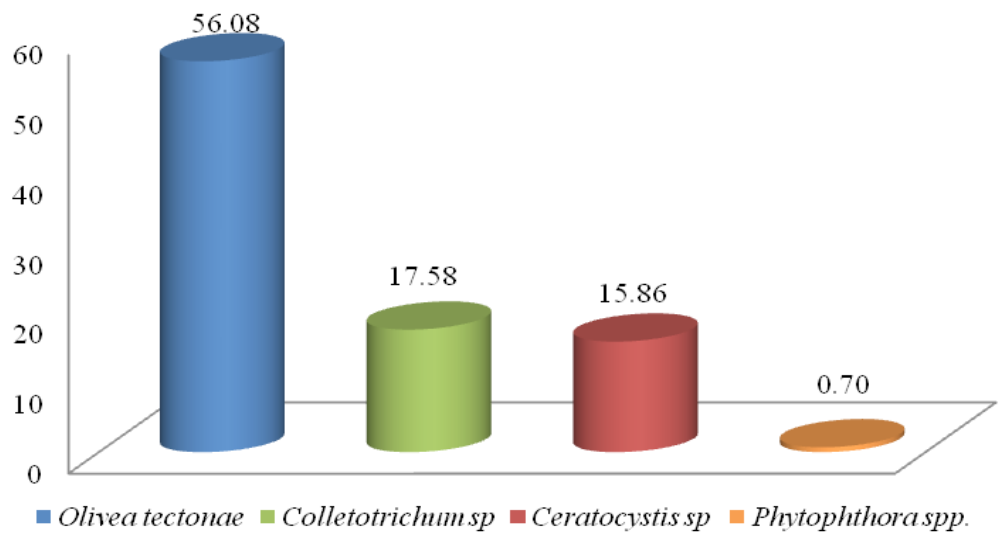

Figura 4. Porcentaje de incidencia de enfermedades causadas por hongos patógenos durante la época seca del año 2004 en cuatro haciendas de la empresa Tropibosques S.A. 


\section{Discusión}

L os resultados de laboratorio reportaron una asociación de escolitidos con Ceratocystis sp. en árboles de Teca, lo que coincide con Cibrián et al. (1995) quien menciona que insectos de la familia Scolytidae como vectores del hongo Ceratocystis sp. en árboles débiles de diferentes especies de Pinos en México.

Los síntomas de Olivea tectonae en la zona de Balzar, provincia del Guayas se comenzaron a evidenciar a inicios del mes de mayo, lo que concuerda con Belezaca (2004) quien reporta la presencia de esta enfermedad en el mismo mes en la zona de Quevedo, provincia de Los Ríos. La presencia de este patógeno en las plantaciones de Ecuador coincide con Arguedas (2004) quien menciona que este patógeno solo había sido detectado en Pakistán e India, antes de presentarse en plantaciones de Teca en Costa Rica y Panamá.

La alta severidad de Olivea tectonae registrada en plantaciones de Teca de 1 a 6 años reportadas en este estudio durante la época seca coincide con lo mencionado por Matarrita (2004) quien reportó una mayor severidad de esta enfermedad en árboles de Teca de edad intermedia de 5 a 7 años en Costa Rica.

En árboles de Teca de un año de edad se evidenció los ataques de barrenadores en la época seca, lo que coincide por lo expresado por el CATIE (1991) y Arguedas (2003) citados por Fonseca (2004) quienes reportaron barrenadores atacando árboles de Teca en Costa Rica, a Phyllophaga sp. atacando las raíces y al hongo Phytophthora spp. causando pudriciones.

La hormiga arriera Atta sp. fue una de las plagas defoliadoras más importantes durante la época seca, lo que coincide por lo expresado por Gara y Onore (1989) y el CATIE (1991) quienes manifiestan que la hormiga arriera es una plaga de importancia en árboles de Teca en Ecuador y Centro América.

La presencia de insectos plagas succionadores de savia, principalmente Oncometopia sp. en la época lluviosa, concuerda por lo expresado por Ford (1981) citado por Madrigal (1986) quien expresa que esta plaga ha sido observada atacando a la Teca y a varias especies forestales, aunque no la considera plaga de importancia forestal.

La presencia de Phyllophaga spp. en árboles de dos a seis años durante la época lluviosa concuerda con lo expresado por Arguedas (2003) quien reporta un fuerte impacto de esta larva en plantaciones recién establecidas y hasta de tres años de edad en Costa Rica.

Algunos insectos y enfermedades encontrados en las plantaciones de Teca de Balzar coinciden con los reportados por Matarrita (2004) quien reporta como plaga de los brotes y el follaje a Atta sp. y como enfermedades a Nigrospora sp. y Olivea tectonae, como enfer- medad del fuste a Botryodiplodia sp. y como plaga de la raíz a Phyllophaga sp. y como enfermedad a Phytophtora spp., en plantaciones de Teca de Costa Rica durante los años 1996 a 2004.

\section{Conclusiones}

$\mathrm{L}^{\mathrm{a}}$ as principales enfermedades presentes en las plantaciones de Teca fueron: Quema de los brotes por Ceratocystis sp., pudrición radicular por Phytophthora sp., marchitez por Colletotrichum sp., roya por Olivea tectonae, secamiento descendente por Botryodiplodia theobromae, marchitamiento del follaje por Pestalotia palmarum.

Los insectos plagas más importantes encontrados en la Teca de la zona de Balzar fueron: Atta sp. (Hymenóptera, Formicidae), Hemileuca maia Drury (Lepidóptera, Saturniidae), Schistocercu spp. (Orthóptera, Acrididae), Scolytus sp. (Coleóptera, Scolytidae), Phyllophaga sp. (Coleóptera, Scarabaeidae), Oncometopia sp. (Homóptera, Cicadellidae), Oberea tripunctata (Coleóptera, Cerambicidae).

En la época lluviosa las enfermedades que tuvieron mayor incidencia fueron el marchitamiento causado por Colletotrichum sp. con $28.16 \%$ y Ceratocystis sp. con $11.86 \%$. Los insectos de mayor incidencia en esta época fueron el pulgón Hyadaphis erysimi (Homóptera, Aphydidae) con 26.07\%, Hortensia similis (Homóptera, Cicadellidae) con 6.80\% y Phyllophaga spp. con $6.20 \%$ (Coleóptera, Scarabaeidae).

Durante la época seca las enfermedades que tuvieron mayor incidencia fueron la roya Olivea tectonae con $56.08 \%$, Colletrotrichum con $17.58 \%$, Ceratocystis sp. con $15.86 \%$ y Phytophthora spp. con $0.70 \%$. Los insectos plagas más importantes en esta época fueron las orugas defoliadoras Hemileuca maia Drury (Lepidóptera, Saturniidae) con 7.96\%, hormigas arrieras Atta sp. (Hymenóptera, Formicidae) con $3.22 \%$ y los perforadores de corteza Scolytus sp. (Coleóptera, Scolytidae) con $2.25 \%$ de incidencia.

Durante la época lluviosa la enfermedad a nivel vascular más representativa fue la causada por Ceratocystis sp., esta enfermedad en la época seca tuvo una mayor severidad en los árboles de Teca.

La barrenación del duramen por Oberea tripunctata (Coleóptera, Cerambicidae) se constituyó en el daño más importante para la Teca, puesto que compromete el valor económico del árbol. 
Flores et al.

\section{Literatura Citada}

Arguedas, M. 2003. Problemas fitosanitarios en Teca (Tectona grandis L.F) en América Central: nuevos reportes. In: Memoria del Seminario y grupo de discusión virtual sobre Teca. Universidad Nacional. Instituto de Investigación y servicios Forestales. Heredia, Costa Rica. 11 p.

Arguedas, M. 2004. La roya de la Teca Olivea tectonae (Rac.): consideraciones sobre su presencia en Panamá y Costa Rica. Kuru Revista Forestal. p 1 -6 .

Belezaca, C. 2004. La roya de la Teca en Ecuador. Plagas Forestales Neotropicales 72: 98 - 99.

CATIE (Centro Agronómico de Investigación y Enseñanza). 1991. Plagas y enfermedades forestales en América Central. Manual de consulta. Turrialba, Costa Rica. 187 p.

Cibrian, D., T. Mendez, R. Campos, H. Yates y J. Flores. 1995. Insectos forestales de México. D. R. Universidad Autónoma de Chapingo. 453 p.

Fonseca, W. 2004. Manual para productores de Teca (Tectona grandis L. F) en Costa Rica. Características y Propiedades de la madera. (en línea). Costa Rica. Consultado el 27 jul. 2004. Disponible en: http://www.fonafifo.com/text_files/proyectos/manualProductoresTeca.pdf

Gara, R. y G. Onofre. 1989. Entomología Forestal. Ministerio de Agricultura y Ganadería. Proyecto DINAF. AID. Quito, Ecuador. 267 p.

Gómez, P. 1999. Problemas fitosanitarios de los cultivos de Maracuyá, Papaya, y Piña en el trópico húmedo del Litoral ecuatoriano. Tesis Ing. Agr. Universidad Técnica de Babahoyo. Babahoyo, Ecuador. p 65.

Madrigal, A. 1986. Inventario de insectos dañinos en los departamentos de Cauca y Valle. In: Revista Miscelánea $\mathrm{N}^{\mathrm{o}}$ 6. Sociedad Entomológica. 30 p.
Matarrita, L. 2004. Prevalencia de la roya de la Teca Olivea tectonae (Rac.), en Costa Rica C.A. Tesina Maestría. Colegio de Posgraduados. Instituto de Fitosanidad. Texcoco, México. 53 p.

MAG (Ministerio de Agricultura y Ganadería). 1986. Inventario de plagas, enfermedades y malezas del Ecuador. Quito, Ecuador. 185p.

OIMT (Organización Internacional de las Maderas Tropicales). 2004. Actualidad Forestal Tropical In: Boletín de la OIMT. Volumen 12. Número 3. 4 p.

Pinzón, O. 1997. Guía de insectos dañinos en plantaciones forestales. CONIF. Santa Fe de Bogotá D.C. Colombia. $99 \mathrm{p}$.

Pinzón, O. 2007. Problemas fitosanitarios en plantaciones forestales en Colombia. Generalidades. (En línea). Colombia. Consultado el 26 de sep del 2008. Disponible en: http:/www.cerambycoidea.com/ titles/pinzonflorian2004.pdf

Ramírez, L. 1997. Guía de enfermedades en plantaciones forestales. CONIF. Santa Fe de Bogotá D.C. Colombia. 49 p.

Rodríguez, R., A. Pérez, P. Posos, J. Martínez, C. Duran y V. Aceves. 2006. Efectividad biológica de fosetil aluminio al $80 \%$ vs Mildew (Pseudoperonospora cubensis) en calabacita. (En línea). Mx. Consultado el 14 de octubre del 2008. Disponible en: http:// www.cucba.udg.mx/new/publicaciones/avances/ avances_2006/Agronomia/RodriguezRuvalcabaRamon/Rodriguez_Ruvalcaba_Ramon.pdf

Weaver, P. L. 2000. Tectona grandis L.F. Teca. Producción de semillas y su diseminación. (En línea). U.S.A. Consultado el 28 de ago. del 2007. Disponible en: http://www.fs.fed.usglobaliitfTectonagrandis.pdf 